\title{
Properties of Stellar Populations in Isolated Lenticular Galaxies
}

\author{
Ivan Yu. Katkov \\ Faculty of Physics, Sternberg Astronomical Institute \\ Lomonosov Moscow State University Moscow, 119991, Russia \\ katkov.ivan@gmail.com \\ Olga K. Sil'chenko \\ Sternberg Astronomical Institute Lomonosov Moscow State University, Moscow, 119991, Russia \\ silesai.msu.su \\ Victor L. Afanasiev \\ Special Astrophysical Observatory of the Russian Academy of Sciences, Nizhnji Arkhyz, 369167 Russia \\ vafan@sao.ru
}

\begin{abstract}
In this paper we present the results of long-slit spectral observations for a sample of isolated lenticular galaxies, made with the SCORPIO and SCORPIO-2 spectrographs of the 6-meter BTA telescope of the SAO RAS. By applying full spectral fitting technique using the stellar population evolutionary synthesis models, we have measured the radial profiles of the stellar line-of-sight velocity as well as the velocity dispersion, SSP-equivalent age and SSP-equivalent metallicity of stars along the radius in 12 targets. The resulting averaged ages of the stellar population in bulges and discs cover an entire range of possible values from 1.5 to $15 \mathrm{Gyr}$ which indicates the absence of a certain formation epoch for the structural components in the isolated lenticular galaxies, unlike in the members of clusters and rich groups: they could have been formed at a redshift of $z>2$ as well as only a few billion years ago. Unlike S0 galaxies in more dense environments, the isolated galaxies typically have the same age of stars in the bulges and discs. The discembedded lenses and rings of increased stellar brightness, identified from the photometry in 7 of 11 galaxies, do not differ strongly from the stellar discs as concerning the properties of stellar populations and stellar velocity dispersion. We conclude that the final shaping of the morphological type of a lenticular galaxy in complete isolation is critically dependent on the possible regimes of cold-gas accretion from outside.
\end{abstract}

Subject headings: galaxies: elliptical and lenticular - galaxies: evolution - galaxies: formation - galaxies: kinematics and dynamics - galaxies: structure.

\section{INTRODUCTION}

The problem of formation and evolution paths of galaxies is the key point in modern extragalactic astronomy and observational cosmology. Galaxies are formed being shaped under a large number of physical factors which are often poorly known. The main issue here is to identify the most important factors which are crucial during the formation and evolution of galaxies of a given morphological type.

The morphological type of lenticular (S0) galaxies was proposed by Edwin Hubble as a hypothetical one in 1936 Hubble (1936) in order to fill in the intermediate position between elliptical and spiral galaxies. It was assumed that the objects of this type had to have large-scale stellar discs as observed in the spiral galaxies but could not demonstrate any noticeable starforming regions and spiral patterns in their stellar discs. Their smooth reddish view and probably an old mean age of stars made them to look similar to the elliptical galaxies. An intermediate position of lenticular galaxies between the purely spheroidal stellar systems and the spiral galaxies, where the contribution of the bulge into the total luminosity decreases monotonically with the transition from early to late types (from left to right along the Hubble sequence of morphological types), gives rise to a natural assumption that the $\mathrm{S} 0$ galaxies should possess large bulges. However, the detailed surface photometry of S0 galaxies has shown that the bulges in them can be either very large or tiny (Laurikainen et al. 2010). Stemming from these results, an old idea of Sidney van den Bergh (1976), proposing that the lenticular galaxies should be forming a sequence parallel to the spiral galaxies in the Hubble fork, and the connection between the (close) position in this diagram of the $\mathrm{S} 0(\mathrm{a}, \mathrm{b}, \mathrm{c})$ and the spiral galaxies of the corresponding subtypes is determined by 
the "bulge/disc" luminosity ratio Kormendy \& Bender 2012; Cappellari et al. 2011), becomes increasingly popular. It would seem that such a turn in the understanding of the Hubble sequence would only reinforce the conventional point of view about the formation of lenticular galaxies via the cessation of star formation in the discs of spiral galaxies: the event of the transformation of a progenitor galaxy into the resulting $\mathrm{S} 0$ galaxy is much easier to imagine when both galaxies have the same bulge-todisc ratio. However, it should be noted that if the contribution of the bulge to the total luminosity in an S0 galaxy is the same as in the spiral galaxy located nearby in the morphological diagram, then this leaves open the possibility of reverse transformation, namely, the transformation of an S0 galaxy into a spiral one, by gas acquisition and subsequent star formation burning, which would have been quite impossible in the presence of a systematically larger bulges in the S0s.

The literature discusses a huge number of physical processes that could cease star formation in a disc of a spiral galaxy. Here there are some of them listed:

- direct collisions of galaxies (Spitzer \& Baade 1951; Icke 1985);

- gravitational tidal effects from the dark halo of the cluster/group (Byrd \& Valtonen 1990);

- "harassment" or the gravitational tidal influence of galaxies on each other during a sufficiently fast flyby (Moore et al. 1996);

- ram pressure of the hot intracluster medium (Gunn \& Gott 1972; Quilis et al. 2000);

- "starvation" or termination of star formation because of disappearance of external reservoirs of gas, previously maintaining the gas accretion onto the disc of a galaxy and feeding its star formation Larson et al. 1980).

These processes are closely related to the density of environments of galaxies, because only the clusters and rich groups of galaxies with their massive dark haloes can provide the necessary density of the hot intergalactic medium for the ram pressure and tight packing of galaxies for the efficiency of gravitational tides.

It is known that lenticular galaxies are the dominant population of nearby clusters of galaxies, where their fraction reaches up to $60 \%$ (Dressler 1980). However, the number of S0 galaxies is quite noticeable among the field galaxies as well: according to the APM survey (Naim et al. 1995), the fraction of lenticular galaxies in the nearby Universe is about 15\%, and they are the second class by the frequency of occurrence after the spirals. Furthermore, we know cases of completely isolated lenticular galaxies (Sulentic et al. 2006). Here we pose a question which has not yet been raised, about the origin of such galaxies. Under which physical mechanisms were they formed, and how do these mechanisms differ from those that work in the dense environment?

Despite the apparent deficit of mechanisms which may be responsible for the morphological transformation of isolated galaxies, in comparison to the members of clusters and groups, it is wrong to suppose that the isolated galaxies evolve completely internally, following the "closed box" scenario. In our work (Sil'Chenko et al. 2011) we have demonstrated that a completely isolated early-type spiral galaxy NGC 7217 over the last 5 Gyr experienced at least two events of minor merging. In a locally isolated S0 galaxy NGC 4124 we have also found signatures of minor merging, which had apparently taken place 2-3 Gyr ago and had provoked a central starburst (Zasov et al. 2013). Furthermore, it was recently understood that lenticular field galaxies often possess significant amounts of gas. Moreover, it is exactly in the rarefied environment that a galaxy often reveals decoupled kinematics of stars and gas, implying external origin of the gas (Davis et al. 2011). Thus, the study of the properties of isolated lenticular galaxies would allow to concentrate on the evolutionary mechanisms, related either to the internal disc instabilities or just the external accretion of gas and/or companion gas-rich galaxies. It should be noted that the accretion of gas and/or minor merging events may not only suppress the star formation in the disc but, on the contrary, stimulate its burst (Birnboim et al. 2007; Sancisi et al. 2008).

The targets studied in this work are the nearby, strongly isolated lenticular galaxies, for which we have fulfiled long-slit spectroscopic observations with the aim to determine the resolved properties of stellar populations. The properties of the ionized gas and its kinematics in comparison to the stellar component kinematics are discussed in the separate paper (Katkov et al. 2013), while in this paper we concentrate only on the ages and chemical composition of the stellar populations.

The paper is organized as follows: Section 2 is devoted to the description of the sample; the details of the spectral observations, reduction, and data analysis are discussed in Section 3. Section 4 presents the results for every galaxy; Sections 5 and 6 contain the discussion of the results and the summary.

\section{SAMPLE SELECTION}

We have compiled a sample of nearby isolated lenticular galaxies by using an approach that has recently been developped in the Laboratory of Extragalactic Astrophysics and Cosmology of the Special Astrophysical Observatory (SAO) RAS by Karachentsev, Makarov, and their co-authors. This approach has been applied by them to the galaxies of the Local Supercluster and its surroundings to extract pairs (Karachentsev \& Makarov 2008), triplets (Makarov \& Karachentsev 2009), and groups (Makarov \& Karachentsev 2011), as well as to select isolated galaxies (Karachentsev et al. 2011). The data on the line-of-sight (LOS) velocities, apparent mag- 
nitudes, and morphological types of the galaxies were taken from the updated HyperLeda database 1 and the NED database 2 supplemented by the velocity measurements from the SDSS, 6dF, HIPASS, and ALFALFA surveys. Clustering was analyzed for the galaxies with the line-of-sight velocities relative to the Local Group of $V_{\mathrm{LG}}<4000 \mathrm{~km} \mathrm{~s}^{-1}$, and for the galaxies within the galactic latitude of $|b|>10 \mathrm{deg}$. A particular benefit of the clustering algorithm proposed by the authors is taking into account the individual characteristics of galaxies, especially, the indicator of the galaxy massthe near-infrared $K$-band luminosity. By linking the galaxies into systems pairwise, the authors suppose that every virtual pair has to satisfy the negative total energy condition and the condition of embedding its components within the "zero velocity sphere", i.e. the galaxies of the pairs do not recede from each other as a result of the Hubble expansion. The clustering algorithm involves an iterative analysis of all galaxies of the original sample for their further integration into the groups or clusters through identifying the bound pairs of galaxies having common members. The details of the algorithm are given in the papers mentioned above. One of the by-products of the whole procedure is a list of pairwise isolation indices calculated between any two galaxies of the sample. The isolation index $(I I)$ of two galaxies is the number characterizing the mutual dynamic influence of components on each other. In the case of an unbound pair, $\log I I$ is positive and equal to the logarithm of the factor by which it is necessary to increase the mass of one of the components so that the pair would meet the given association criteria. And conversely, in the case of a bound pair, $\log I I$ is negative and equal to the logarithm of the factor by which the mass has to be reduced to become unbound. The same value of the isolation index may be found for a wide pair of galaxies of comparable luminosities and for a tight pair consisting of a giant galaxy and a close faint companion.

The authors of this approach have kindly provided us with the information about the mutual isolation indices for all galaxies of the Local Supercluster and its environs. By using this information, we have selected for our study the lenticular galaxies having $I I>2.5$ for the galaxies of higher and lower luminosity if compared to the considered one.

We have made deep long-slit spectroscopic observations of 12 galaxies from the resulting list in order to study the properties of their stellar populations. Table 1 lists five the most important neighbours of higher and lower luminosity for every galaxy observed by us so far as well as the information about the morphology, line-ofsight velocities, and absolute magnitudes. In the cases of the NGC 16 and NGC 3098 galaxies, the conditions on the isolation from the potential companions of lower luminosities are not satisfied. Both galaxies have low-

${ }^{1}$ http://leda.univ-lyon1.fr/

2 http://ned.ipac.caltech.edu/ luminosity neighbours with $I I=1.9$; but due to a very large luminosity difference, $\delta M_{K} \approx 5$ (the masses differ by a factor of nearly 100), we believe that the companions cannot have a significant gravitational effect on large galaxies, so these galaxies do not egress from the generally accepted selection criteria for isolated galaxies.

\section{OBSERVATIONS AND DATA ANALYSIS}

\subsection{Observations}

The observations of the sample of isolated lenticular galaxies were carried out in the prime focus of the $6-\mathrm{m}$ Russian telescope of the SAO RAS over the period of 2011-2012. The spectral data for all the galaxies, except NGC 6615 and NGC 6654, were obtained with the SCORPIO-2 focal reducer (Afanasiev \& Moiseev 2011) in the long-slit mode with the slit width of 1 arcsec and the slit length of 6.1 arcminutes. For the observations we have used the VPHG 1200@540 - volume-phase holographic grating (the grism), which provides a spectral resolution of $\mathrm{FWHM} \approx 4 \AA$ in the range of $3800-7300 \AA$. This spectral range includes both strong absorption lines, like $\mathrm{Mg}, \mathrm{Fe}, \mathrm{G}-\mathrm{band}$, and a number of ionized-gas emission lines $(\mathrm{H} \alpha, \mathrm{H} \beta,[\mathrm{O} \mathrm{III}],[\mathrm{NII}]$, etc.) that allows to explore both the kinematics, age, and chemical composition of the stellar component and the kinematics and excitation of the ionized gas. We used the CCD detector E2V 42-90 with the chip size $2 \mathrm{k} \times 4 \mathrm{k}$ which at the $1 \times 2$ binning mode readout provides a spatial scale along the slit of 0.357 per pixel and reciprocal dispersion of $0.86 \AA$ per pixel. Unlike the other galaxies, NGC 6615 and NGC 6654 were observed with another instrumental configuration, namely, with the SCORPIO instrument Afanasiev \& Moiseev 2005) and the VPHG 2300G grism that provided a spectral resolution of $2.2 \AA$ in the range of 4800-5600 $\AA$, and the use of the EEV CCD 42-40 detector with the chip size $2 \mathrm{k} \times 2 \mathrm{k}$ gave the same scale along the slit with the reciprocal dispersion of $0.37 \AA$ per pixel. During all the observations the slit was aligned along the outer-isophote major axes of the galaxies. The log of the observations (Table 2) gives the dates of observations, total exposures, average seeing conditions during the exposure of every galaxy, and the position angle of the slit.

\subsection{Primary data reduction}

Primary data reduction was performed by using the original programs, developed in the IDL environment, including the following steps: taking into account the registration system bias by subtracting the averaged zero exposure frame from all the images; taking into account the uneven illumination and inhomogeneities in the CCD sensitivity through dividing the spectra by the flat-field calibration lamp; removal of the traces of cosmic hits by using the L.A.Cosmic algorithm van Dokkum 2001), which implements the Laplacian filter for hit trace detection, and addition of spectra after cleaning; twodimensional wavelength calibration by using the spectrum of the helium-neon-argon lamp and the further lin- 


\begin{tabular}{|c|c|c|c|c|c|c|c|c|c|c|c|c|}
\hline \multirow{2}{*}{ Galaxy } & \multicolumn{2}{|c|}{ Type } & \multirow{2}{*}{$V_{s y s}$} & \multirow{2}{*}{$M_{K}$} & \multicolumn{4}{|c|}{ Upper galaxies } & \multicolumn{4}{|c|}{ Downer galaxies } \\
\hline & HyperLEDA & NED & & & Galaxy & $\delta M_{K}$ & $\delta V$ & $\Pi I$ & Galaxy & $\delta M_{K}$ & $\delta V$ & $I I$ \\
\hline \multirow{5}{*}{ IC0875 } & \multirow{5}{*}{ So } & \multirow{5}{*}{ S0 } & \multirow{5}{*}{2913} & \multirow{5}{*}{-22.713} & NGC4814 & 1.034 & 278 & 31.0 & PGC046033 & -3.949 & -48 & 4.2 \\
\hline & & & & & NGC5218 & 1.175 & -121 & 107.9 & SDSSJ1324... & -3.577 & -126 & 10.3 \\
\hline & & & & & NGC5322 & 2.242 & 993 & 126.9 & NGC4964 & -0.851 & 268 & 57.3 \\
\hline & & & & & NGC5430 & 1.491 & -224 & 150.4 & NGC5109 & -1.563 & 660 & 78.6 \\
\hline & & & & & UGC08237 & 0.315 & -86 & 179.2 & SDSSJ1259... & -4.268 & -83 & 84.0 \\
\hline \multirow{5}{*}{ IC1502 } & & & & & IC0356 & 1.793 & 1131 & 158.6 & UGC12247 & -4.960 & 298 & 46.2 \\
\hline & S0-a & $\mathrm{S}^{\wedge}+$ & 2237 & -23.704 & NGC3031 & -0.394 & 2135 & 280.1 & UGC12504 & -4.322 & -461 & 64.6 \\
\hline & & & & & NGC1184 & 0.925 & -264 & 311.8 & UGC12921 & -2.427 & -477 & 73.5 \\
\hline & & & & & IC0342 & -0.361 & 1997 & 376.8 & UGC12160 & -1.135 & 404 & 74.2 \\
\hline & & & & & NGC6951 & 1.074 & 517 & 473.3 & UGC12069 & -2.052 & -405 & 106.8 \\
\hline & & & & & NGC7817 & -0.190 & 745 & 214.2 & PGC000446 & -5.443 & -140 & 1.9 \\
\hline NGC0016 & E-S0 & $\mathrm{SABO}^{\wedge}-$ & 3300 & -24.511 & NGC0253 & -0.100 & 3024 & 1029.7 & UGC12873 & -5.043 & -212 & 22.8 \\
\hline & & edge-on & & & NGC3031 & -1.201 & 3198 & 1212.8 & PGC087206 & -5.736 & -513 & 191.4 \\
\hline & & & & & NGC7619 & 1.071 & -689 & 1296.4 & PGC001153 & -6.733 & -636 & 312.1 \\
\hline & & & & & NGC7331 & 0.410 & 2179 & 1339.5 & UGC00285 & -3.498 & 872 & 459.0 \\
\hline & & & & & NGC2339 & 1.168 & -396 & 135.1 & UGC03775 & -4.638 & -244 & 12.1 \\
\hline NGC2350 & S0-a & $\mathrm{S} 0 / \mathrm{a}$ & 1774 & -22.725 & NGC2365 & 0.681 & -429 & 400.0 & UGC03691 & -0.708 & -327 & 81.7 \\
\hline & & & & & NGC3031 & 0.584 & 1672 & 402.6 & PGC097214 & -2.601 & -298 & 102.4 \\
\hline & & & & & NGC4472 & 2.552 & 901 & 635.7 & PGC2807004 & -5.452 & 40 & 172.1 \\
\hline & & & & & IC0342 & 0.618 & 1534 & 1054.5 & - & -5.371 & -154 & 470.6 \\
\hline & & & & & NGC3190 & 1.456 & 117 & 4.1 & PGC2806869 & -4.981 & 58 & 1.9 \\
\hline NGC3098 & S0-a & S0 & 1305 & -22.170 & NGC3245 & 1.216 & 29 & 27.1 & IC2520 & -1.545 & 151 & 24.1 \\
\hline & & edge-on & & & NGC3227 & 0.955 & 271 & 40.4 & UGC05588 & -2.090 & 95 & 33.0 \\
\hline & & & & & NGC2964 & 1.033 & 44 & 49.0 & PGC029347 & -2.527 & 10 & 37.5 \\
\hline & & & & & NGC3379 & 1.618 & 544 & 53.3 & NGC3026 & -1.433 & -109 & 38.5 \\
\hline & & & & & NGC3190 & 1.808 & 168 & 2.5 & PGC166105 & -5.029 & 252 & 13.2 \\
\hline NGC3248 & So & So & 1356 & -21.818 & NGC3301 & 0.782 & 116 & 6.3 & PGC2806870 & -5.424 & -115 & 16.0 \\
\hline & & & & & NGC3245 & 1.568 & 80 & 15.9 & PGC030270 & -3.603 & -78 & 24.1 \\
\hline & & & & & NGC3227 & 1.307 & 321 & 21.4 & UGC05588 & -1.738 & 145 & 35.6 \\
\hline & & & & & NGC3379 & 1.970 & 594 & 38.5 & PGC031387 & -2.524 & 47 & 36.9 \\
\hline & & & & & NGC6574 & 0.520 & 396 & 21.7 & UGC11214 & -1.687 & 34 & 2.5 \\
\hline NGC6615 & $\mathrm{SO}-\mathrm{a}$ & $\mathrm{SBO}^{\wedge}+?$ & 2868 & -23.779 & UGC11057 & -0.299 & -161 & 92.4 & PGC061685 & -2.895 & -198 & 2.9 \\
\hline & & & & & NGC6548 & 0.244 & 498 & 110.9 & PGC061658 & -1.804 & -319 & 13.5 \\
\hline & & & & & NGC6501 & 0.432 & -224 & 123.7 & UGC11168 & -0.981 & 381 & 22.4 \\
\hline & & & & & NGC6587 & 0.390 & -429 & 138.0 & PGC061621 & -0.776 & -274 & 29.4 \\
\hline & & & & & NGC6643 & -0.203 & 459 & 21.1 & PGC062387 & -3.539 & 101 & 18.4 \\
\hline NGC6654 & SO-a & $\left(\mathrm{R}^{\prime}\right) \mathrm{SB} 0 / \mathrm{a}(\mathrm{s})$ & 2204 & -23.830 & NGC6340 & -0.651 & 757 & 189.7 & NGC6654A & -3.756 & 380 & 25.6 \\
\hline & & & & & NGC6951 & 0.948 & 484 & 225.3 & UGC10892 & -4.568 & 26 & 33.3 \\
\hline & & & & & NGC3031 & -0.520 & 2102 & 273.3 & PGC062173 & -3.925 & 534 & 51.3 \\
\hline & & & & & NGC6911 & 0.517 & -576 & 470.4 & UGC11295 & -4.629 & -427 & 57.8 \\
\hline & & & & & NGC6764 & -0.001 & 41 & 31.1 & UGC11457 & -4.740 & -10 & 6.1 \\
\hline NGC6798 & So & So & 2741 & -23.520 & NGC6824 & 1.934 & -1091 & 232.1 & NGC6757 & -0.813 & 75 & 36.9 \\
\hline & & & & & NGC6703 & 1.045 & 90 & 344.4 & UGC11502 & 0.047 & -335 & 112.3 \\
\hline & & & & & NGC6829 & 0.376 & -853 & 505.2 & PGC063313 & 0.604 & -1241 & 193.8 \\
\hline & & & & & NGC6946 & 0.083 & 2389 & 577.0 & NGC6796 & -0.424 & 263 & 303.2 \\
\hline & & & & & NGC0253 & 3.488 & 801 & 137.0 & PGC069415 & -1.826 & 61 & 2.5 \\
\hline NGC7351 & So & $\mathrm{SAB}^{\wedge} 0 ?$ & 1077 & -20.923 & NGC7727 & 3.637 & -881 & 899.2 & PGC069224 & -1.777 & -7 & 3.4 \\
\hline & & & & & NGC3031 & 2.387 & 975 & 1006.1 & PGC1028063 & -3.405 & 23 & 11.9 \\
\hline & & & & & IC1459 & 4.208 & -599 & 1042.6 & PGC982181 & -5.031 & -37 & 169.8 \\
\hline & & & & & NGC7507 & 3.518 & -558 & 1163.6 & PGC069293 & 0.579 & -809 & 626.0 \\
\hline & & & & & NGC2712 & 0.334 & -50 & 66.2 & UGC04659 & -2.959 & 17 & 26.7 \\
\hline UGC04551 & So & S0? & 1794 & -22.633 & NGC2768 & 1.827 & 328 & 69.3 & PGC023834 & -3.120 & -10 & 27.5 \\
\hline & & & & & NGC2639 & 2.259 & -1458 & 71.1 & UGC0 4543 & -1.791 & -189 & 58.5 \\
\hline & & & & & NGC3031 & 0.677 & 1691 & 127.7 & UGC04648 & -3.851 & -138 & 93.2 \\
\hline & & & & & NGC2681 & 0.009 & 1048 & 159.4 & UGC04922 & -1.653 & -228 & 168.9 \\
\hline & & & & & NGC4472 & 3.567 & 909 & 88.7 & NGC5727 & -2.425 & 206 & 42.1 \\
\hline UGC09519 & So & $\mathrm{S} 0 ?$ & 1782 & -21.710 & NGC5353 & 3.466 & -457 & 131.0 & PGC2080256 & -3.641 & -196 & 69.1 \\
\hline & & & & & NGC5611 & 0.804 & -243 & 152.5 & PGC052694 & -2.617 & 132 & 72.0 \\
\hline & & & & & NGC5582 & 0.986 & 247 & 159.8 & UGC09597 & -3.886 & -30 & 123.5 \\
\hline & & & & & NGC5194 & 2.284 & 1221 & 194.7 & NGC5798 & -0.596 & -88 & 127.6 \\
\hline
\end{tabular}

Table 1: Environmental properties of galaxies. 
earization of spectra with the typical accuracy of $0.03-$ $0.06 \AA$ depending on the grism used; subtraction of the background spectrum of the night sky; conversion of the instrumental fluxes into the absolute ones by using the spectra of spectrophotometric standards. During the observations with the Moon high enough the night sky contribution is large and varies with time. In such cases we performed the night sky subtraction before the frame additions to fit the best parameters of the sky background for each spectrum individually. Among the final results of the primary data reduction there were not only the reduced spectra of the objects but also the frames of the errors that were calculated assuming the Poisson photon statistics and readout noise and pushed through all reduction steps together with the data frames.

In addition to the spectra of objects and spectrophotometric standards, the spectra of dawn or twilight sky were also analyzed in the process of data reduction, which, in the essence, represent the solar spectrum dispersed by the Earth atmosphere which are convoluted with the instrumental profile of the spectrograph in every point of the slit. Therefore, the analysis of the spectra of the dawn or twilight sky allows to determine the behaviour of the instrumental profile of the spectrograph along the slit and along the direction of dispersion. The former is important to subtract correctly the contribution of the night sky, and the latter allows to accurately determine the kinematical parameters. The spectra of the dawn sky were reduced in the same manner as the spectra of the galaxies though without the sky background subtraction. The reconstruction of the instrumental profile and its use when analyzing the galaxies are described in detail in the following Section 3.3.

\subsection{Subtraction of night sky contribution}

When analyzing spectral data for objects with low surface brightness, special attention should be paid to the careful subtraction of the contribution of the nightsky background, underestimation of which may lead to systematic errors in the derived parameters of stellar

\begin{tabular}{lcccr}
\hline Name & Date & $\begin{array}{c}\text { Exposure, } \\
\text { s }\end{array}$ & $\begin{array}{c}\text { Seeing, } \\
\text { arcsec }\end{array}$ & $\begin{array}{r}\text { PA, } \\
\text { deg }\end{array}$ \\
\hline IC 875 & Apr 23, 2012 & 2700 & 2.5 & -30 \\
IC 1502 & Nov 19, 2011 & 2700 & 2.5 & 52 \\
NGC 16 & Nov 20, 2011 & 1800 & 2.0 & 16 \\
NGC 2350 & Dec 13, 2012 & 6000 & 1.6 & -73 \\
NGC 3098 & Apr 18, 2012 & 5400 & 1.2 & -90 \\
NGC 3248 & Apr 22, 2012 & 2700 & 3.0 & -45 \\
NGC 6615 & Sep 19, 2012 & 7200 & 1.0 & -15 \\
NGC 6654 & Sep 20, 2012 & 6600 & 1.3 & 0 \\
NGC 6798 & Nov 20, 2011 & 5400 & 2.5 & -30 \\
NGC 7351 & Nov 19, 2011 & 3600 & 2.0 & 0 \\
UGC 4551 & Dec 12, 2012 & 8400 & 2.0 & -67 \\
UGC 9519 & Apr 24, 2012 & 4500 & 2.0 & -105 \\
\hline
\end{tabular}

Table 2: The long-slit observation log. populations (Katkov \& Chilingarian 2011). In this paper we determine the properties of stellar populations in the galactic structural components, including the largescale stellar discs of low surface brightness. Therefore, we believe it is necessary to describe in detail the refined procedures of the night-sky spectrum subtraction.

We have earlier proposed a refined technique for subtracting the spectrum of the night sky for the long-slit spectral data of the low surface brightness objects in case of strong variations of the instrumental profile along the slit Katkov \& Chilingarian 2011). In that approach the sky model is constructed by interpolating the spectrum of the sky from the edges of the slit to the central part containing the spectrum of the object, by applying the deconvolution procedure to the reference spectrum. The spectrum of the twilight sky, which carries the information on the variations of the instrumental profile, is supposed to be used as a reference spectrum. Unfortunately, during the real observations the spectra of the dawn/twilight sky were not routinely observed due to a sudden worsening of weather conditions during the nights. The standard method which does not require the use of the reference spectrum and provides zero approximation for the profile variations along the slit, consists in the approximation of the sky spectrum by the low-power polynomials $(n=2--4)$ in each column of the spectrum image parallel to the slit, and its recomputation for the area of the object. This method works well for the spectra of non-extended objects. However, this straightforward approach is not always suitable for the long-slit observations of galaxies.

Therefore, here we propose another way to build the $2 \mathrm{D}$ spectrum of the night sky, which is based on the extrapolation procedure in the frequency domain. The spectrum of the night sky at a given position on the slit $y-S(\lambda, y)$ can be written as a convolution of the "intrinsic" spectrum of the night sky $S_{0}(\lambda)$ with the instrumental profile $\mathcal{L}(\lambda, y)$ :

$$
S(\lambda, y)=S_{0}(\lambda) \mathcal{L}(\lambda, y)
$$

In the frequency domain the convolution procedure becomes a multiplication. Hence, if we perform a onedimensional Fourier transform along the direction of dispersion, then

$$
\operatorname{FFT}[S(\lambda, y)]=\operatorname{FFT}\left[S_{0}(\lambda)\right] \operatorname{FFT}[\mathcal{L}(\lambda, y)] .
$$

As the analysis of the spectra of the dawn and twilight sky has shown, the shape of the instrumental profile of the SCORPIO/SCORPIO-2 spectrographs varies rather monotonously along the slit, therefore its Fourier transform $\operatorname{FFT}[\mathcal{L}(\lambda, y)]$ varies along the slit monotonously as well. The first factor in the Fourier transform of the night sky spectrum is a constant function, hence in general the Fourier transform of the night sky along the slit varies monotonously. Using this fact and applying the standard polynomial extrapolation procedure to the spectrum of the night sky in the frequency domain, i.e., 
to its Fourier transform, and performing the subsequent inverse Fourier transform, we can construct the model of the spectrum of the night sky. The Fourier transform of the spectral image returns complex values, this is why the extrapolation should be performed separately for the real and imaginary parts of the Fourier transform. In this model of the night sky, the reference spectrum of the dawn/twilight sky is not required. At the same time the quality of the model is comparable to the method based on the deconvolution procedure (Katkov \& Chilingarian 2011).

\subsection{Data analysis}

Before undertaking the analysis of the spectra of galaxies, we analyzed the spectrum of the dawn sky observed the same observational run, in order to estimate the variations of the parameters of the spectrograph instrumental profile (LSF-Line Spread Function) along and across the direction of dispersion which is required to determine properly the internal kinematics of stars and ionized gas in the galaxies. To do this, we have split the frame of the twilight sky spectrum into many areas: 32 intervals along the slit and 7 segments along the dispersion. In every section the twilight sky spectra were co-added to achieve the typical signal-to-noise ratio $S / N=100$ per pixel. Then, we fitted the spectra from every area by the highresolution solar spectrum, taken from the ELODIE 3.1 stellar spectral library (Prugniel et al. 2007), by applying the PPXF procedure of the per-pixel approximation of spectra (Cappellari \& Emsellem 2004). During fitting the twilight sky spectrum, the instrumental profile is parametrized by the Gauss-Hermite series of orthogonal functions (van der Marel \& Franx 1993). As a result of approximation, we derive the instrumental profile (LSF) parameters for different spectral ranges and along the slit. The mean characteristic widths of the instrumental profile (in terms of velocity dispersion) for the observational mode with the VPHG 2300G and VPHG 1200@540 grisms are $\sigma_{\text {instr }}=65 \mathrm{~km} \mathrm{~s}^{-1}$ and $\sigma_{\text {instr }}=90 \mathrm{kms}^{-1}$ respectively.

The further analysis consisted of fitting the observed absorption-line spectra of galaxies by the high-resolution models of stellar populations. To do this, we used the NBuRsts software package (Chilingarian et al. 2007a b), which represents an extension of the PPXF per-pixel spectrum approximation method Cappellari \& Emsellem 2004). The approach applied allows to derive the information about the stellar component from all the available spectral elements in total, in contrast to the analysis of the Lick indices of individual lines (Worthey et al. 1994; Jones \& Worthey 1995) - the indicators of the properties of stellar populations. The approach of per-pixel approximation of the spectra of galaxies allows to exclude from the analysis the narrow spectral ranges around strong emission lines of ionized gas, it allows to avoid their systematic effect on the estimates of the stellar population parameters which is impossible when analyzing the Lick index $\mathrm{H} \beta$. Chilingarian et al. (2007a, b) have shown that the per-pixel spectrum approximation method provides also a higher accuracy, by a factor of $1.5-2$, for the stellar population parameters derived, in comparison to the approach of the Lick indices.

The core of the procedure of determining the parameters of the stellar populations represents nonlinear minimization of the quadratic difference $\left(\chi^{2}\right)$ between the observed and model spectra. We have used synthetic spectra of stellar populations as model spectra. They were computed with the PEGASE.HR Le Borgne et al. 2004 ) evolutionary code based on the ELODIE 3.1 highresolution stellar spectral library (Prugniel et al. 2007) for the simple star formation history in the form of one brief burst (SSP, Simple Stellar Population). The parameters of the SSP stellar population model are the age of the star formation burst $T$ (Gyr) and the metallicity $[\mathrm{Z} / \mathrm{H}]$ (dex), while the Salpeter initial stellar mass function (Salpeter 1955) and solar chemical element ratios are considered to be fixed in the model. To measure the stellar kinematics of the galaxy, line-of-sight velocity distributions (LOSVDs), which are parametrized as the Gauss-Hermite quadrature van der Marel \& Franx 1993), are convolved with the model spectrum. Furthermore, the multiplicative pseudo-continuum is included into the model that allows to take into account the effects of the intrinsic interstellar extinction of a galaxy as well as the possible errors of the flux absolute calibration both in the observational data and in the stellar library, serving as the base on which the stellar population models were synthesized. To take into account the effect of the spectrograph LSF on the spectrum of a galaxy, before fitting the observed spectra we convoluted the grid of stellar population models with the previously determined instrumental profile. The presence of even weak emission lines and/or the residues from the inaccurate subtraction of the strongest night-sky lines can bias the stellar population parameter estimates. Hence, to eliminate this effect, we have masked the spectral ranges of $10-15 \AA$ around them. As a result of the $\chi^{2}$ minimization, the following set of parameters is derived: the line-of-sight stellar velocity $v\left(\mathrm{~km} \mathrm{~s}^{-1}\right)$, the line-of-sight stellar velocity dispersion $\sigma\left(\mathrm{km} \mathrm{s}^{-1}\right)$, the estimates of the SSP-equivalent stellar age $T$ (Gyr) and metallicity $[\mathrm{Z} / \mathrm{H}]$ (dex).

Our spectroscopy was carried out with a long slit, which was aligned along the major axis of the galaxies under consideration. Since the surface brightness of galactic discs decreases with the distance from the center, the spectra of the outer regions of the galaxies have low $S / N$ ratio. To increase it, we used the adaptive binning procedure, by co-adding the spectra over the intervals of variable length along the slit, arranged so that the $S / N$ ratio is to be not less than the preassigned value (typically about 20-30) in each interval.

After subtracting the stellar component model from the observed spectrum of a galaxy, we got a pure emission-line spectrum of the galaxy. Every line was 


\begin{tabular}{|c|c|c|c|c|c|c|}
\hline Galaxy & $\begin{array}{l}\text { Averaging range, } \\
\text { arcsec }\end{array}$ & $\begin{array}{c}\text { Number } \\
\text { of measurements }\end{array}$ & $\begin{array}{l}T, \\
\text { Gyr }\end{array}$ & $\begin{array}{l}{[\mathrm{Z} / \mathrm{H}],} \\
\operatorname{dex}\end{array}$ & $\begin{array}{c}\mid \mathrm{Mg} / \mathrm{Fe}] \\
\mathrm{dex}\end{array}$ & $\begin{array}{c}\sigma, \\
\mathrm{km} \mathrm{s}^{-1}\end{array}$ \\
\hline \multicolumn{7}{|c|}{ Bulge } \\
\hline $\mathrm{IC} 875$ & $4-7$ & 10 & $4.3^{ \pm 0.7}$ & $-0.16^{ \pm 0.05}$ & $0.20^{ \pm 0.04}$ & $110^{ \pm 9}$ \\
\hline IC 1502 & $4-7$ & 11 & $17.6^{ \pm 0.9}$ & $-0.04^{ \pm 0.06}$ & $0.3^{ \pm 0.1}$ & $168^{ \pm 16}$ \\
\hline NGC 16 & $2-5$ & 10 & $5.4^{ \pm 0.8}$ & $-0.04^{ \pm 0.05}$ & $0.19^{ \pm 0.04}$ & $172^{ \pm 6}$ \\
\hline NGC 2350 & $4-7$ & 8 & $1.6^{ \pm 0.3}$ & $-0.13^{ \pm 0.08}$ & - & $103^{ \pm 15}$ \\
\hline NGC 3098 & $4-7$ & 8 & $5.4^{ \pm 0.4}$ & $-0.10^{ \pm 0.02}$ & $0.00^{ \pm 0.02}$ & $73^{ \pm 6}$ \\
\hline NGC 3248 & $4-7$ & 10 & $4.8^{ \pm 0.6}$ & $-0.11^{ \pm 0.05}$ & $0.00^{ \pm 0.05}$ & $77^{ \pm 5}$ \\
\hline NGC 6615 & $4-7$ & 8 & $10.8^{ \pm 1.5}$ & $-0.26^{ \pm 0.05}$ & $0.24^{ \pm 0.03}$ & $129^{ \pm 5}$ \\
\hline NGC 6654 & $2-5$ & 9 & $12.2^{ \pm 1.4}$ & $-0.19^{ \pm 0.07}$ & $0.23^{ \pm 0.04}$ & $158^{ \pm 5}$ \\
\hline NGC 6798 & $4-7$ & 6 & $8.0^{ \pm 1.9}$ & $-0.20^{ \pm 0.05}$ & $0.13^{ \pm 0.04}$ & $115^{ \pm 7}$ \\
\hline NGC 7351 & $4-7$ & 10 & $2.2^{ \pm 0.5}$ & $-0.37^{ \pm 0.08}$ & $-0.03^{ \pm 0.06}$ & $29^{ \pm 11}$ \\
\hline UGC 4551 & $4-7$ & 8 & $10.0^{ \pm 1.9}$ & $-0.28^{ \pm 0.08}$ & $0.15^{ \pm 0.03}$ & $158^{ \pm 11}$ \\
\hline UGC 9519 & $4-7$ & 9 & $2.5^{ \pm 0.1}$ & $-0.12^{ \pm 0.06}$ & $0.04^{ \pm 0.03}$ & $76^{ \pm 3}$ \\
\hline \multicolumn{7}{|c|}{ Disc } \\
\hline $\mathrm{IC} 875$ & $13-45$ & 16 & $2.9^{ \pm 0.9}$ & $-0.32^{ \pm 0.18}$ & $0.26^{ \pm 0.07}$ & $134^{ \pm 27}$ \\
\hline IC 1502 & $7-25$ & 11 & $16.7^{ \pm 1.6}$ & $-0.13^{ \pm 0.10}$ & $0.42^{ \pm 0.01}$ & $130^{ \pm 25}$ \\
\hline NGC 16 & $6-30$ & 18 & $1.6^{ \pm 1.2}$ & $-0.19^{ \pm 0.15}$ & $0.16^{ \pm 0.02}$ & $127^{ \pm 18}$ \\
\hline NGC 2350 & $10-40$ & 15 & $1.3^{ \pm 0.2}$ & $-0.00^{ \pm 0.07}$ & $0.06^{ \pm 0.07}$ & $86^{ \pm 14}$ \\
\hline NGC 3098 & $25-60$ & 18 & $5.1^{ \pm 1.5}$ & $-0.22^{ \pm 0.06}$ & $0.08^{ \pm 0.02}$ & $57^{ \pm 26}$ \\
\hline NGC 3248 & $10-39$ & 31 & $3.9^{ \pm 1.4}$ & $-0.21^{ \pm 0.09}$ & $-0.04^{ \pm 0.03}$ & $65^{ \pm 17}$ \\
\hline NGC 6615 & $40-60$ & 0 & - & - & - & - \\
\hline NGC 6654 & $35-60$ & 3 & $5.8^{ \pm 0.6}$ & $-0.06^{ \pm 0.14}$ & $0.40^{ \pm 0.20}$ & $44^{ \pm 5}$ \\
\hline NGC 6798 & $8-55$ & 18 & $7.3^{ \pm 4.3}$ & $-0.27^{ \pm 0.15}$ & $0.11^{ \pm 0.12}$ & $119^{ \pm 17}$ \\
\hline NGC 7351 & $17-45$ & 7 & $4.4^{ \pm 2.3}$ & $-0.57^{ \pm 0.19}$ & $-0.02^{ \pm 0.15}$ & $72^{ \pm 40}$ \\
\hline UGC 4551 & $38-80$ & 3 & $10.9^{ \pm 4.3}$ & $-0.74^{ \pm 0.39}$ & $0.25^{ \pm 0.25}$ & $107^{ \pm 20}$ \\
\hline UGC 9519 & $16-30$ & 4 & $2.9^{ \pm 0.9}$ & $-0.32^{ \pm 0.17}$ & $0.15^{ \pm 0.20}$ & $98^{ \pm 15}$ \\
\hline \multicolumn{7}{|c|}{ Lens/Ring } \\
\hline IC 875 & - & 0 & - & - & - & - \\
\hline IC 1502 & - & 0 & - & - & - & - \\
\hline NGC 16 & $12-21$ & 16 & $3.3^{ \pm 2.9}$ & $-0.25^{ \pm 0.16}$ & - & $104^{ \pm 16}$ \\
\hline NGC 2350 & $20-26$ & 1 & $4.9^{ \pm 0.0}$ & $-0.33^{ \pm 0.00}$ & - & $97^{ \pm 0}$ \\
\hline NGC 3098 & $15-20$ & 14 & $4.8^{ \pm 1.3}$ & $-0.13^{ \pm 0.05}$ & $0.05^{ \pm 0.01}$ & $57^{ \pm 12}$ \\
\hline NGC 3248 & - & 0 & - & - & - & - \\
\hline NGC 6615 & $20-40$ & 3 & $12.8^{ \pm 2.4}$ & $-0.52^{ \pm 0.16}$ & $0.21^{ \pm 0.06}$ & $56^{ \pm 5}$ \\
\hline NGC 6654 & - & 0 & - & - & - & - \\
\hline NGC 6798 & $14-22$ & 10 & $5.2^{ \pm 2.1}$ & $-0.30^{ \pm 0.14}$ & $0.13^{ \pm 0.04}$ & $97^{ \pm 15}$ \\
\hline NGC 7351 & - & 0 & - & - & - & - \\
\hline UGC 4551 & $17-35$ & 12 & $3.3^{ \pm 2.2}$ & $-0.47^{ \pm 0.23}$ & $0.23^{ \pm 0.03}$ & $117^{ \pm 25}$ \\
\hline UGC 9519 & $7-15$ & 22 & $2.7^{ \pm 0.5}$ & $-0.22^{ \pm 0.07}$ & $0.05^{ \pm 0.02}$ & $77^{ \pm 9}$ \\
\hline
\end{tabular}

Table 3: The mean stellar population parameters of the bulges, discs, and lenses/rings 
approximated by a Gaussian to obtain the line fluxes, velocities, and velocity dispersions of ionized gas. In this paper we only consider the properties of the stellar populations, while the paper (Katkov et al. 2013) is dedicated to the properties of the ionized gas in these galaxies.

The PEGASE.HR stellar population models were synthesized basing on the library of spectra of stars in the solar vicinity, possessing the solar $\alpha$-element-to-iron abundance ratio. The resulting models are hence only computed for the solar abundance ratios. The methods for constructing the stellar population models taking into account non-solar $\alpha$-element abundances are currently being developed (see, e.g., the studies (Walcher et al. 2009) and (Prugniel \& Koleva 2012)). However, these first-trial models are calculated for a rather rare and limited grid of parameters and are so far inferior in quality to the models with the solar abundance ratio. The relative abundance of $\alpha$-elements bears information on the duration of the main star formation epoch which has provided the bulk of stars. If the burst was very brief, shorter than $1 \mathrm{Gyr}$, then the stellar populations would demonstrate an excess of the $\alpha$-elements with respect to iron in comparison with the solar chemical composition. Given a long history of star formation, as in the Sun's environs, the ratio of abundances of iron and $\alpha$-elements, magnesium in particular, becomes solar (Tinsley 1979; Matteucci \& Greggio 1986). To estimate the relative abundance of $\alpha$-elements of the stellar populations in the galaxies under consideration, in addition to the per-pixel approximation method we have also undertaken classical Lick-index approach. For the spectra of the galaxies observed we have calculated the Lick $\mathrm{H} \beta$, Mgb, Fe 5270, and Fe 5335 indices, the definition of which were taken from (Worthey et al. 1994; Jones \& Worthey 1995). By comparing the obtained indices to the model values, calculated in the framework of stellar population synthesis models for different values of magnesium-to-iron ratios (Thomas et al. 2003), we have derived the $\mathrm{Mg} / \mathrm{Fe}$ ratio values.

\section{RESULTS}

Fig. 1 presents the derived radial profiles of the stellar component properties for each studied galaxy: the line-of-sight velocity profile of stars $v$, stellar velocity dispersion $\sigma$, and the properties of the stellar populations: the SSP-equivalent age $T$ and the SSP-equivalent metallicity $[\mathrm{Z} / \mathrm{H}]$. In addition, we have analyzed the available photometric data for these galaxies and have identified the areas where the disc starts to dominate in the total surface brightness, where the more complex morphological structures such as rings and lenses are present, and where in the center of a galaxy the region of the bulge prevalence can be distinguished (while the nucleusdominated region is excluded). For this purpose, we have used data from the public archive of the SDSS survey, DR9, in the $r$ filter (for the most galaxies), or, if some galaxies are not observed in the SDSS survey, we have used the 2MASS survey data, summed in the $J, H, K$ filters (for NGC 2350, NGC 6798, IC 1502), and also the white-light images obtained with the SCORPIO-2 in the imaging mode during the spectral observations of NGC 6798 and IC 1502. For the NGC 6798 and IC 1502 the image-analysis results have coincided in the near infrared bands and in the white light. For every galaxy we have fulfilled an isophote analysis and then have treated the profiles of azimuthally averaged surface brightness. The outer regions being well described by an exponential law of the surface brightness decrease outward where also constant ellipticity of the isophotes is observed have been considered as the disc dominance regions. The local brightness excesses are sometimes visible in the surfacebrightness profiles, which we identify as rings or lenses. To estimate the bulge parameters, we have used a fixed range of radii, $4^{\prime \prime}-7^{\prime \prime}$. Over the radial ranges restricted in this way, we have made the averaging of the stellar population parameters taking the weights inversely proportional to the squares of the individual estimate errors, $w_{i}=1 / \delta p_{i}^{2}$. The mean parameter estimates for the bulges, discs, and rings or lenses are listed in Table 3. A comparison of the derived parameters is also plotted graphically in Figs. 2, 3, and 4,

\subsection{IC 875}

In the HyperLeda and NED databases this galaxy is classified as a lenticular, and hence we have included it into our sample. However, after the analysis of the spectral data, we have found that it proved to possess rather slow rotation. If we adopt the inclination as $i=50^{\circ}$ from the NED, then $V_{\text {rot }}=\Delta V_{\mathrm{LOS}} / \sin i \approx 65 \mathrm{~km} \mathrm{~s}^{-1}$, while the stellar velocity dispersion is measured to be $\sigma \approx 110 \mathrm{kms}^{-1}$, that gives the ratio of the rotation velocity to the velocity dispersion $V_{\max } / \sigma \approx 0.6$. The ratio of the major and minor axes, taken from NED, gives the isophote ellipticity of $\epsilon=0.25$. These values put the galaxy at the Binney's diagram $\left(V_{\max } / \sigma-\epsilon\right)$ onto the line of isotropic spheroids supported by rotation; this dynamical status is also common for low-luminosity elliptical galaxies (Davies et al. 1983). So we conclude that IC 875 is probably an elliptical galaxy. We hence eliminated it from our sample of isolated lenticular galaxies, and it was not used to construct diagrams and distributions over the parameters for the isolated S0 galaxies.

\subsection{IC 1502}

In the NED, this galaxy is classified as $\mathrm{S}^{+}$. It has a rather large inclination $i=64^{\circ}$, according to the HyperLeda. The radial line-of-sight velocity profile reveals regular rotation. The main feature of this galaxy is that having the solar metallicity, the SSP-equivalent age of the stars is about 15-17 Gyr over the whole measured radial range. The fact that this age has appeared larger than the cosmological age of the Universe should not be confusing. The main input components of the evolutionary synthesis models of the stellar populations are isochrones of stars, which come from the stellar evolution theory and 

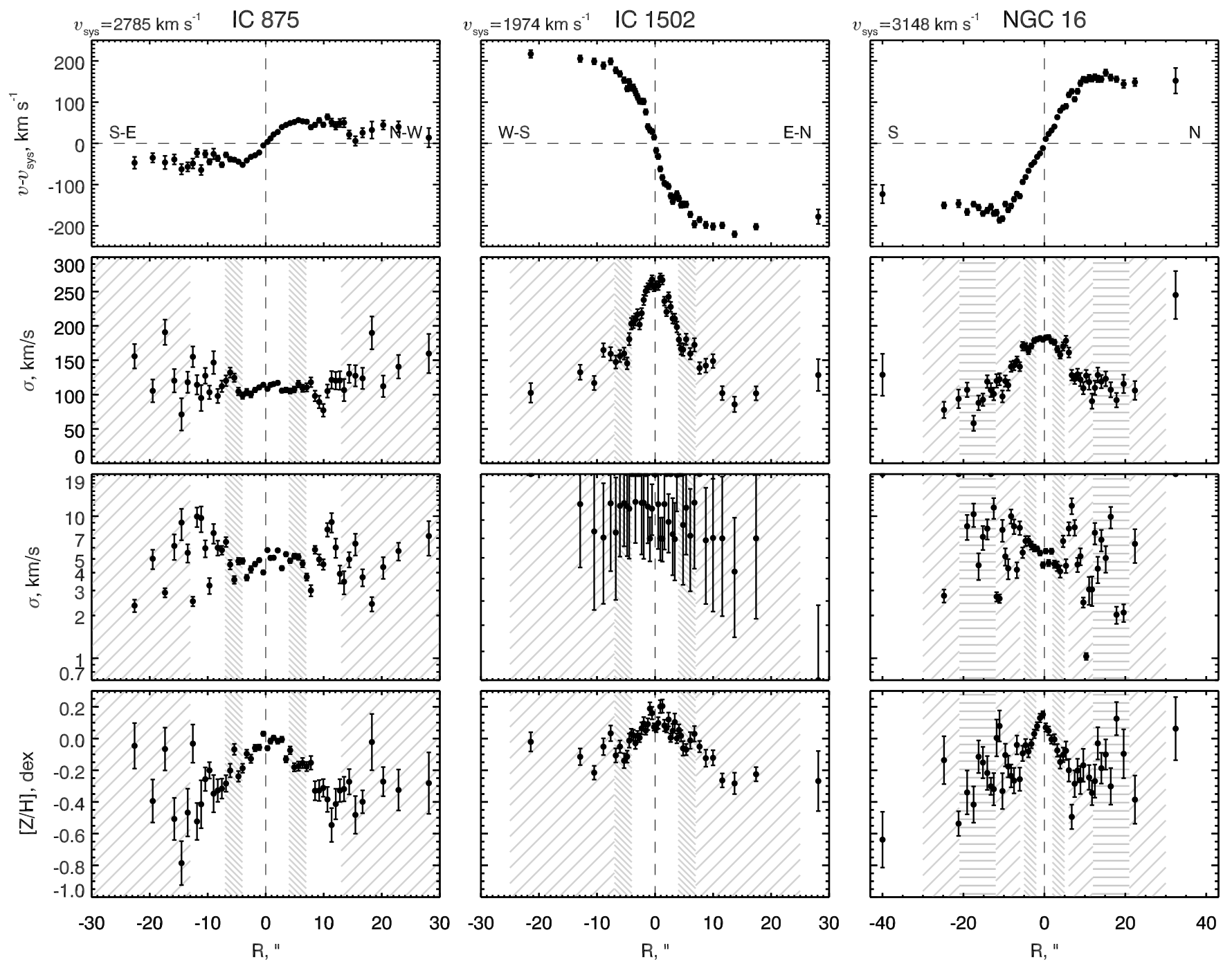

Fig. 1.- The results of analysis of the long-slit spectra of the galaxies under consideration. Each column corresponds to one galaxy. The panels with radial profiles of the line-of-sight velocity of stars after subtracting the systematic velocity, stellar velocity dispersions, ages, and metallicities are plotted from top to bottom. The distance from the center of a galaxy in arcseconds is plotted along the $x$-axis. The gray slashes mark the areas of the averaging of the bulge parameters $(\backslash)$ and the exponential disc parameters $(/)$, the horizontal lines $(-)$ are the regions of the lens or the ring dominance, if present. The systematic velocity corresponds to the measured line-of-sight velocity of the galaxy's center, i.e., the brightest part, except for IC 875 where this is the center of symmetry of the central part of the velocity profile. 

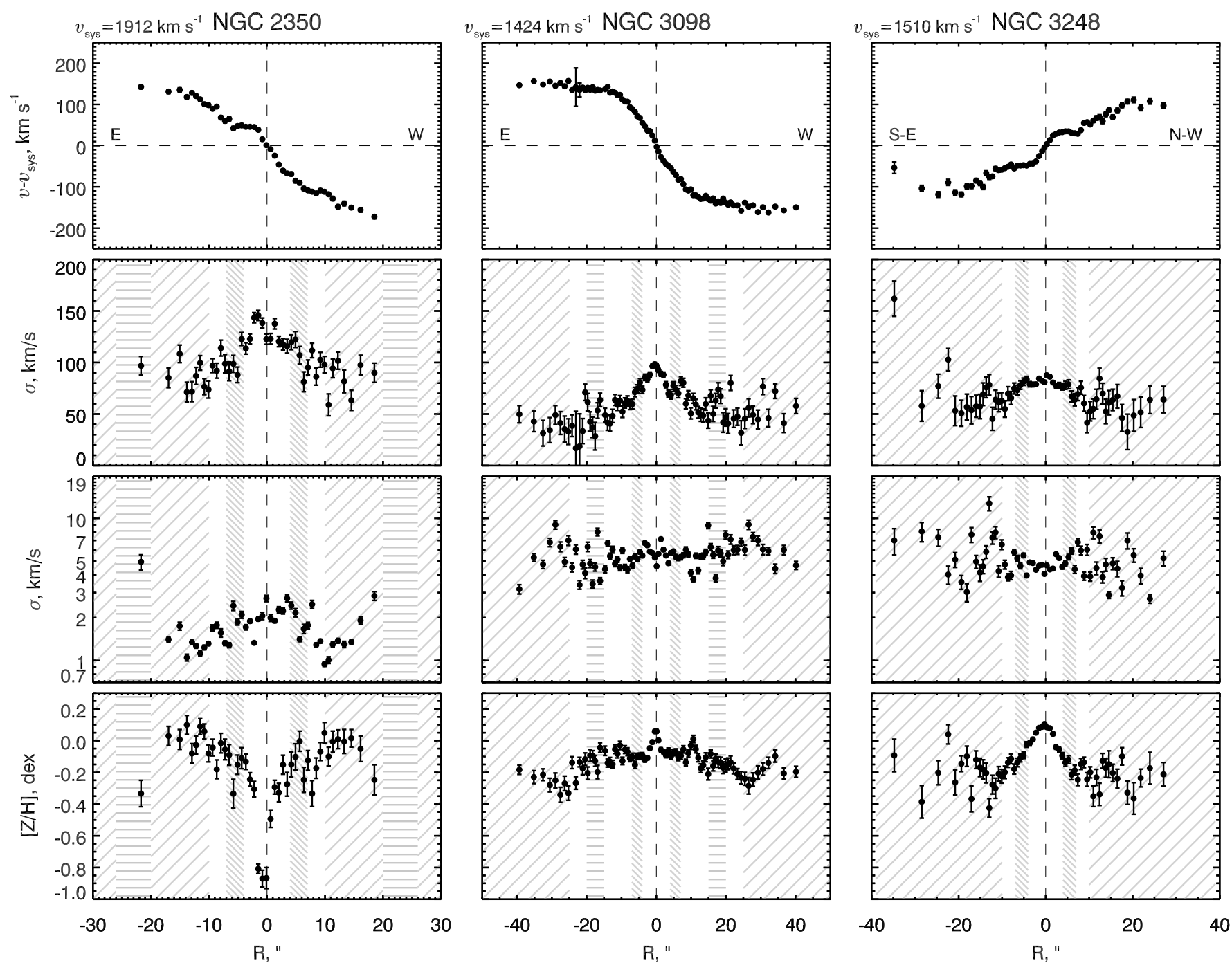

Fig. 1.-(Cont.) 

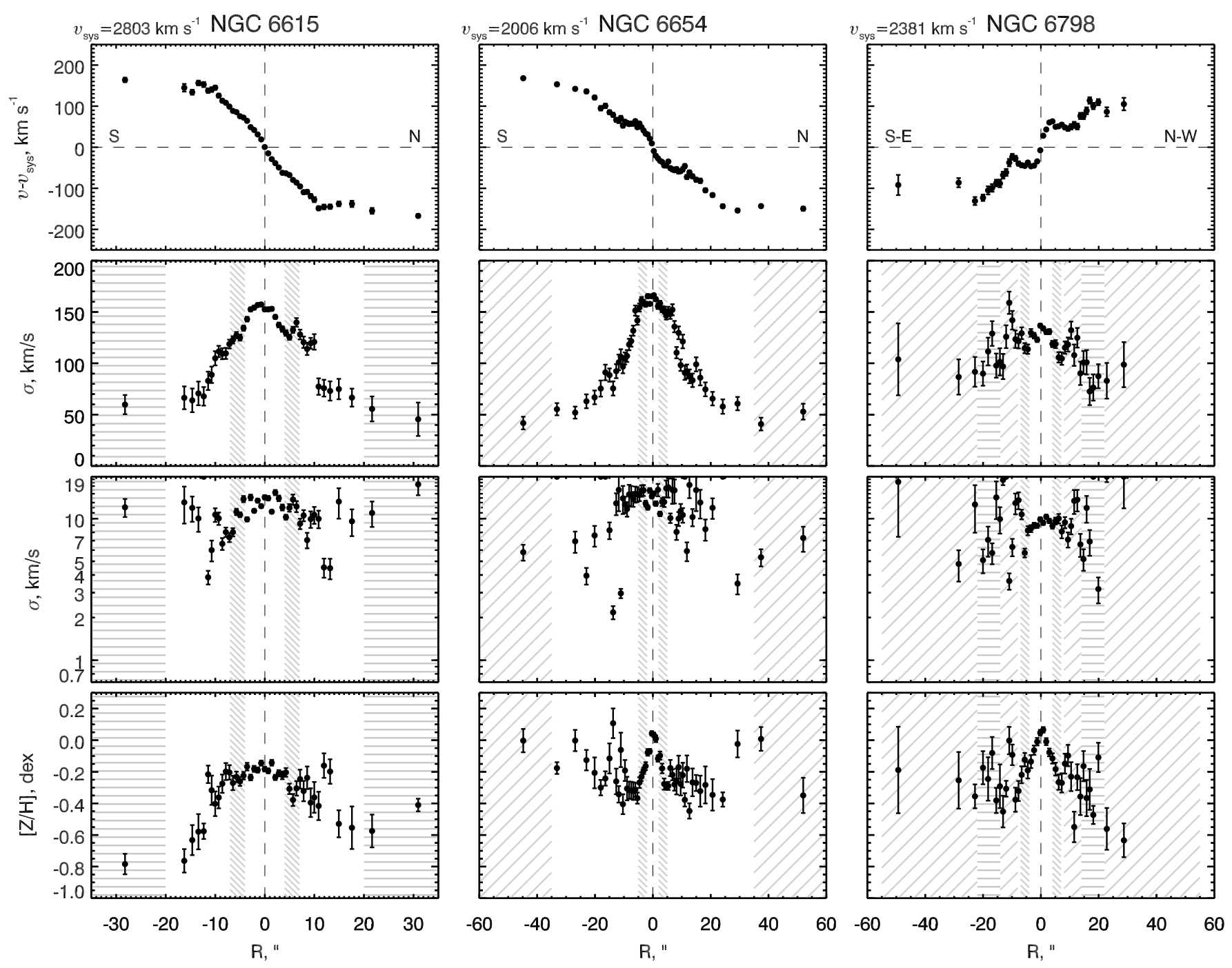

Fig. 1.-(Cont.) 

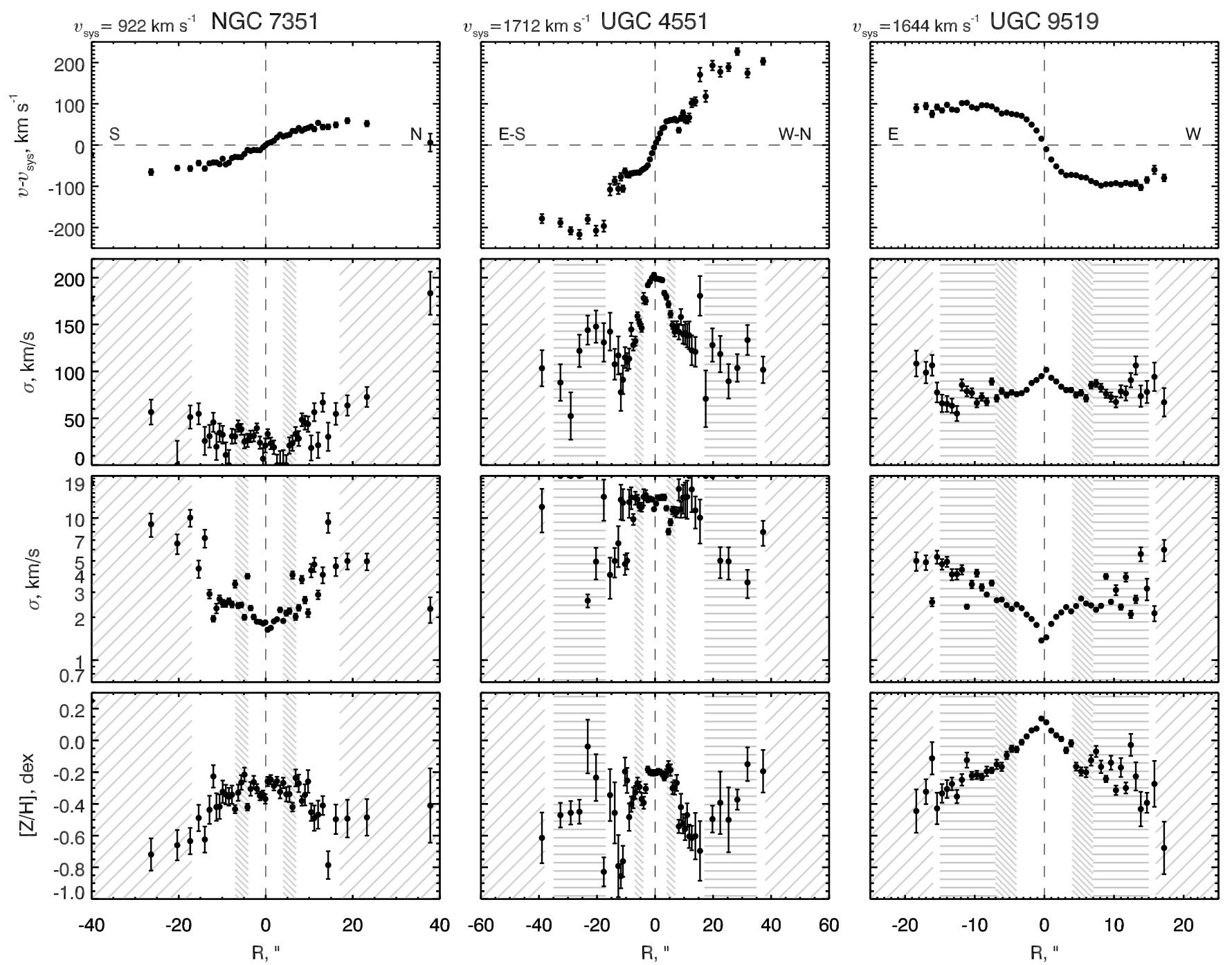

Fig. 1.-(Cont.) 
from the empirical library of stellar spectra. It should be borne in mind that timescales of the stellar evolution used so far have nothing in common with the cosmological models of the Universe and provide absolutely independent channel of information about the age of the dominant stellar populations in galaxies. The radial profiles of the stellar parameters in IC 1502 demonstrate the central kinematically detached region, which betrays itself as a steep stellar velocity gradient and a small decrease in the stellar velocity dispersion. The metallicity profile reveals a marginal dip. All the combination of the features is probably the evidence of evolutionary isolation of the nuclear star cluster, although as for the age of the nuclear stellar population it does not stand away within the measurement error.

\subsection{NGC 16}

This galaxy has a small bar aligned at a right angle to the line of nodes of the galactic disc and a faint latetype companion, with the magnitude difference toward the host of $\Delta m_{B}=4^{\mathrm{m}}$, Barway et al. 2005) at $500 \mathrm{kpc}$ from it. The isolation index between the companion and the host galaxy is $I I=1.9$, but since the mass difference is about two orders of magnitude, we consider NGC 16 as a quite isolated from the external tidal effects. The galaxy has an extended rigid-body rise of the rotation curve of up to $10^{\prime \prime}$ from the center, where the rotation velocity reaches a plateau, and its stellar disc is rather dynamically hot, $\sigma>100 \mathrm{~km} \mathrm{~s}^{-1}$. The stellar component in NGC 16 has an intermediate age, the bulge and the disc being of similar ages in the mean, although individual age estimates in the bins demonstrate large scatter.

\subsection{NGC 2350}

This poorly studied galaxy was unfortunately missed by the SDSS survey. According to the HyperLeda and $\mathrm{NED}$, it is classified as $\mathrm{S} 0 / \mathrm{a}$. In the white-light image obtained with the SCORPIO-2 at the $6 \mathrm{~m}$ Russian telescope, we see a complex distribution of surface brightness shaped as bright spots at the edges of the bar - the socalled "ansae" phenomenon. In addition, from the analysis of the emission-line component of the spectrum, the galaxy has an extended disc of ionized warm gas. Plotting the emission-line ratios onto the diagnostic BPTdiagrams, we have made sure that it is excited mainly by the radiation of young stars (Katkov et al. 2013); i.e., this lenticular galaxy is undergoing current star formation over the entire disc. Correspondingly, the averaged age of the stars within the area bordered by the "ansae" is pretty young: individual estimates in the bins oscillate between 1 and 2 Gyr. In the central region of the galaxy the stars demonstrate a very low metallicity. This may be due to a previous event of a metal-poor companion infall. At the same time this region distinguishes itself in the rotation curve and at the velocity dispersion profile, but, to our surprise, it does not stand out at the age profile. According to our estimates, the metallicity of the gas is only slightly subsolar even in the regions with the very low stellar metallicity. Therefore, the ionized gas in this galaxy is probably not related by its origin to a possible merger having produced the nuclear stellar cluster.

\subsection{NGC 3098}

It is a well-known isolated, edge-on lenticular galaxy. Its photometric structure was studied by Seifert \& Scorza 1996). It was noted that a bulge of the galaxy was small and compact, and between the bulge and the disc, approximately at the radius of $15^{\prime \prime}$, there was a ring of enhanced stellar surface brightness. Our results (Fig. 1) confirm the small effect of the bulge- the rotation velocity growth in the center of the galaxy is very slow-and demonstrate a homogeneous age of $5-8$ billion years for the stellar populations across the whole galaxy as well as a sharp drop of the stellar metallicity in the transition from the central part of the galaxy to the region dominated by the large-scale stellar disc. The stellar disc looks dynamically cold, $\sigma<60 \mathrm{~km} \mathrm{~s}^{-1}$.

\subsection{NGC 3248}

Beyond a radial distance of $100 \mathrm{kpc}$, the galaxy is surrounded by a dozen of faint companions, the brightest of which is only $3^{\mathrm{m}}$ fainter than the host galaxy. The central region of the galaxy, $R<15^{\prime \prime}$, has been investigated in detail by means of panoramic spectroscopy with the SAURON spectrograph within the ATLAS-3D survey Cappellari et al. 2011; Davis et al. 2011). The galaxy is found to contain a lot of gas, ionized, neutral and molecular, and all this gas counterrotates with respect to the stellar component. We have expanded the kinematic profiles to the distance of $30^{\prime \prime}$ from the center and confirmed the counterrotation of the gas concentrated in the central region of the galaxy. The ionized-gas excitation is shock-like, and there are no signs of ongoing star formation. The age of the stellar populations both in the center and in the galactic disc is intermediate, but the central regions are significantly more metal-rich than the disc.

\subsection{NGC 6615}

This galaxy is classified as barred in all the extragalactic databases. Visual inspection of the SDSS images confirms that a compact bar is present and oriented almost orthogonally to the major axis of the galactic disc. The surface brightness profile reveals a noticeable extended stellar lens with a flat brightness distribution, while the exponential disc itself starts at the radii greater than $40^{\prime \prime}$. The sensitivity of our spectroscopy with the highresolution VPHG2300 holographic grating (the grism) was not enough to reach the disc, and we have identified the characteristics of the stellar populations only in the bulge and in the lens. The age of the stellar population is uniformly old throughout the entire studied part of the galaxy. It is possible that between the bulge/bar and the lens a relatively young narrow stellar ring is present. 

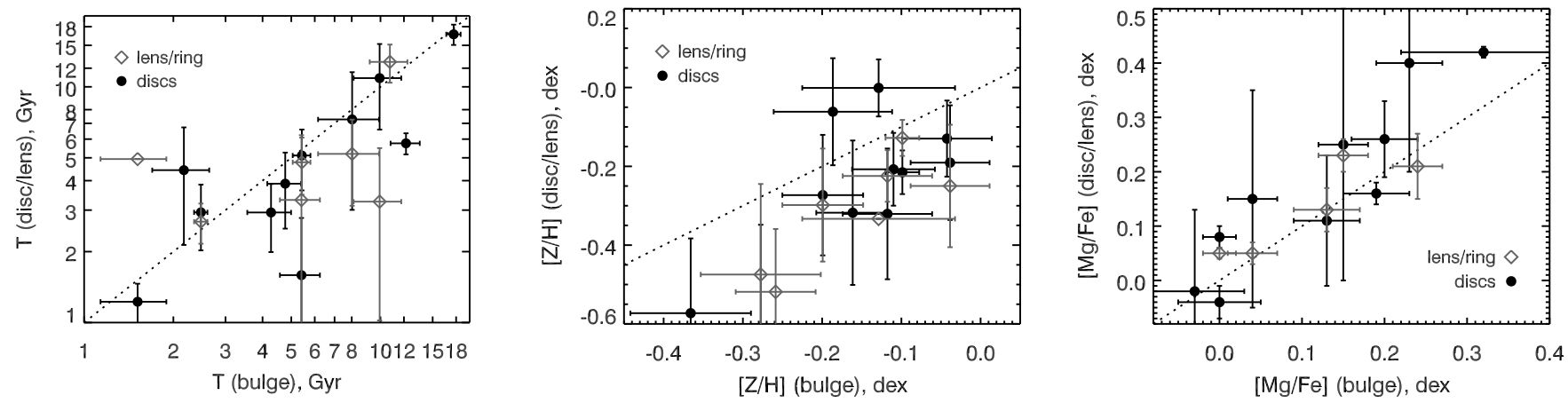

Fig. 2.- A comparison of parameters of the stellar populations in the bulges with the properties in the discs and the disc components: lenses/rings. The dashed line marks the line of equality.
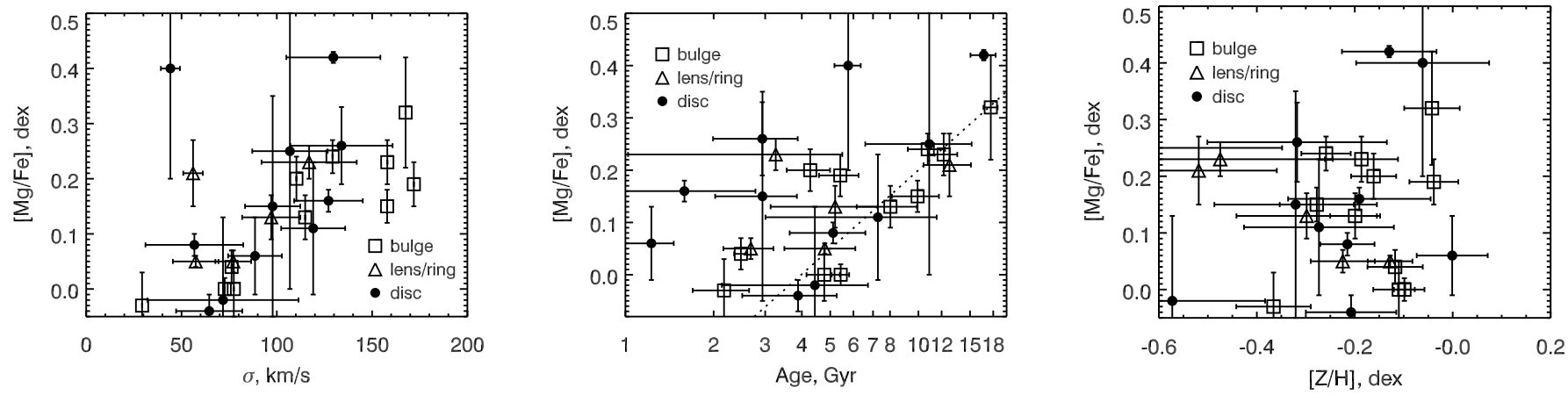

Fig. 3.- A comparison of the velocity dispersion, ages, and metallicities with the relative enrichment of the $\alpha$ elements $([\mathrm{Mg} / \mathrm{Fe}])$ for different structural components of the galaxies.

The metallicity is below the solar everywhere, while in the lens it is significantly lower than solar, at least by a factor of 3-4. The emission-line gas in the galaxy is not detected, but the stellar lens is dynamically cold.

\subsection{NGC 6654}

It is a luminous galaxy with a large-scale bar and a large-scale stellar disc of low surface brightness. The NED database gives it a classification as $\left(\mathrm{R}^{\prime}\right) \mathrm{SB}(\mathrm{s}) 0 / \mathrm{a}$. We have accepted the main parameters of the photometric structure of the galaxy from (Laurikainen et al. 2010). The outer edge of the disc reveals an emissionline gaseous ring with ongoing star formation. Apart from this outer ring, the ionized gas is only present in the central region of the galaxy, where it demonstrates shock-like excitation and rotates too fast to be in the main symmetry plane of the galaxy which is inclined by some $45^{\circ}$ to the line of sight (according to the HyperLeda). In our work (Katkov et al. 2013) we speculated that the central gas rotates in the plane inclined to the main symmetry plane of the galaxy. The characteristics of the stellar population (Fig. (1) indicate that the disc is younger and richer by metals than the bulge. Keeping in mind the residual star formation at the periphery of the disc, we can conclude that NGC 6654 is a rare case of a lenticular galaxy, where rejuvenation (or secondary star formation) has taken place in the disc and not in the central region. Moreover, given the noticeable overabundance of the magnesium relative to the iron in the stellar disc (Table 3), this rejuvenation has had character of a very brief starburst provoked by expanding density wave.

\subsection{NGC 6798}

It is another galaxy which was studied earlier within the ATLAS-3D survey (Cappellari et al. 2011; Davis et al. 2011), like NGC 3248, and where a counterrotating disc of the ionized gas was found, while the neutral hydrogen in this galaxy is extended far beyond the stellar disc. Analyzing the photometric structure of the galaxy with the white-light image obtained by using the SCORPIO-2, we have noted the presence of a stellar ring at the radius of $15^{\prime \prime}-20^{\prime \prime}$. This ring is $5 \pm 2$ Gyr old that, taking into account the error bar, almost coincides with the age of the disc of $7 \pm 4 \mathrm{Gyr}$. However the ring is slightly cooler dynamically than the disc that is consistent with its per- 

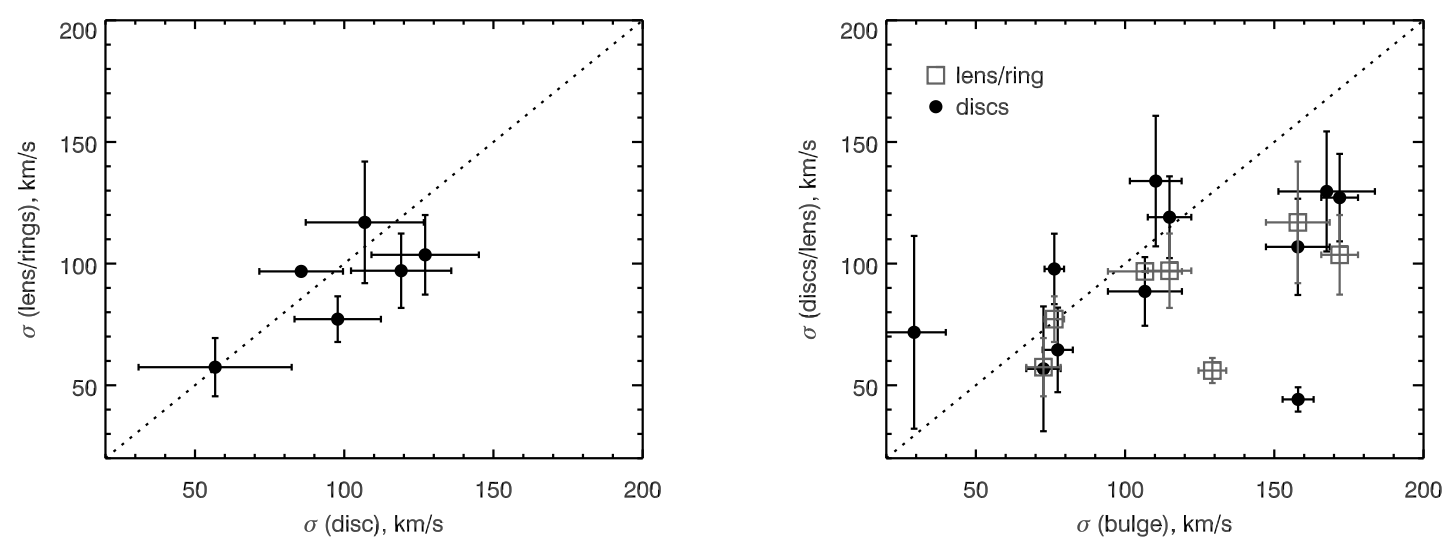

Fig. 4.- Left: a comparison of the stellar velocity dispersion in the disc and in separate structures-rings or lenses. Right: a comparison of the stellar velocity dispersion in the bulge and in the disc structures.

haps younger age. At the same time the metallicity of the stars throughout the galaxy is homogeneous, being half-solar.

\subsection{NGC 7351}

It is a dwarf lenticular galaxy with a low stellar velocity dispersion both in the center and in the disc, and with a slow rotation velocity. Its stellar kinematics was previously investigated by (Simien \& Prugniel 2002). As it might be expected in the low-luminosity galaxy, the metallicity of its stars is below the solar one, but while in the center it is lower than the solar only by a factor of 2.5 , in the disc it is lower at least by a factor of 4. The ages of the stellar population monotonically increase from the center to the edge of the galactic disc, from 1.5 Gyr in the nucleus to 5-8 Gyr in the disc. The comparison with the characteristics of the gaseous component which we published in (Katkov et al. 2013) has shown that in the center the ionized gas rotates together with the stars and is excited by current star formation, while outside the central region the gas distribution deviates from the plane of the galactic disc, and its kinematics becomes strongly decoupled from the stellar kinematics. In this case the accretion of external gas has obviously taken place from a highly inclined orbit, and due to this inclination stationary conditions for star formation (rejuvenation) have emerged only in the center of the galaxy, where the gas has accumulated and come to the galactic symmetry plane.

\subsection{UGC 4551}

It is one more galaxy with a counterrotating gaseous component, which may rotate towards the stars right in the plane of the galactic disc (Katkov et al. 2013). Nevertheless, both the central part of the galaxy and its external disc contain uniformly old, $T>10 \mathrm{Gyr}$, stellar populations (Fig. 1). A stellar lens with a flat brightness profile is observed between the bulge and the disc. Here, in the lens, stellar population is significantly younger than in the disc and in the bulge. From the dynamical point of view both the lens and the disc look rather "hot" $\sigma>100 \mathrm{~km} \mathrm{~s}^{-1}$.

\subsection{UGC 9519}

This galaxy, although having an almost face-on orientation with respect to the line of sight $\left(i=23^{\circ}\right.$, HyperLeda), demonstrates very rapid apparent rotation of the stars (Fig. 1). We (Katkov et al. 2013) noted earlier strong divergency of the kinematics of the stars and ionized gas in our long-slit data. Panoramic spectral data of the ATLAS-3D survey Cappellari et al. 2011; Davis et al. 2011) gives rather evidence for the inner polar gaseous disc in this galaxy. As in the case of NGC 7351, the SSP-equivalent age of the stars monotonically rises along the radius of the galaxy, from $1.5 \mathrm{Gyr}$ in the nucleus to about 5 Gyr in the disc. The disc, being the oldest stellar component of the galaxy, is also the most dynamically hot, $\sigma \approx 100 \mathrm{kms}^{-1}$. It is also metal-poor, about twice poorer than the solar chemical composition. In the center of the galaxy the metallicity gets slightly higher than the solar one, that may be understood given recent additional star formation activity here.

\section{DISCUSSION}

Here we wish to compare the stellar population properties of the different structural components of the galaxies: the comparison of the mean ages will help to trace a sequence of basic evolutionary stages in the life of a galaxy, while the magnesium-to-iron abundance ratio will allow to estimate the typical duration of the main events of star formation in various components of a galaxy. The main cosmological paradigm to date, the LCDM model of the evolution of the Universe, predicts that the classical spheroidal bulges of the early- 
type disc galaxies are the first to form in a major merging event, and only after their shaping the large-scale discs have to emerge around them by a smooth accretion of cold gas and subsequent star formation. However, there are many data which contradict these predictions: again and again, the imaging surveys of galaxies demonstrate a correlation between the metric scales of the bulges and the discs. Interestingly, this correlation is always found independently on whether the given sample of galaxies is populated by classical or pseudobulges (MacArthur et al. 2003; Méndez-Abreu et al. 2008; Laurikainen et al. 2010). Thus, it looks like the formations of bulges and discs in galaxies are synchronized.

This is exactly what we see in Fig. 2 The comparison of the mean ages of the stellar populations in the bulges and in the flat components, namely, in the discs and lenses, shows that our galaxies are concentrated to the bisector, i.e., on the average the ages of the stellar populations in the bulges and in the discs are the same. There are only two objects in which the stellar age of the disc is certainly less than the stellar age of the bulge. This is a particular feature as concerning the evolution of our isolated lenticular galaxies: at the same diagram for the S0 galaxies in denser environments, studied by Sil'chenko et al. (2012), the objects are assembled in the left upper corner, above the bisector, i.e., the ages of the discs are typically either equal or older compared with the bulge stellar ages. We expected such difference between the S0 galaxies in groups and in isolation: all the known mechanisms, both gravitational and gasdynamical, of the external influence on a galaxy are associated with dense environment and lead to "inflow" of the gas into galactic center, thus provoking a secondary star formation burst exactly in the central areas, in the regions dominated by the bulges (Bekki \& Couch 2011; Kronberger et al. 2008). Interestingly, the bulges and discs have appeared to possess equal magnesium-to-iron abundance ratios. This means that either the star formation ends quickly in both structures or that it goes on for billions of years here and there. This result, namely, the similarity of both the mean ages and the $\mathrm{Mg} / \mathrm{Fe}$ ratios in the bulges and discs allows us to strengthen the conclusion on the synchronous formation of bulges and discs: the star formation in both components starts and ends quasi-simultaneously. Despite this, the mean metallicity of stars in the discs is significantly lower than that in the bulges. Does that mean that the accretion of metalpoor gas occurs mainly to the external discs, while the "fuel" for star formation in the bulges comes there after its enrichment by the heavy elements in the discs?

Fig. 3 compares $\mathrm{Mg} / \mathrm{Fe}$ ratio, characterizing the duration of the main starforming episode, with the other characteristics of the stellar populations for all three types of structural components. Again, we see an impressive synchronizing of the evolution of the bulges and the discs: through all the relations, the signs marking the different structural components are uniformly mixed in the graphs. The correlation of the stellar magnesium-to-iron ratio with the stellar velocity dispersion, characterizing the local gravitational potential, is well-known among elliptical galaxies and bulges (Trager et al. 2000) and is considered to be a proof of connection between the star formation efficiency and the potential well depth. However, the left panel of Fig. 3reveals for the first time that this correlation takes also place among the discs of isolated lenticular galaxies, and it looks even tighter than the one for the bulges. By analogy, we can suppose that the deeper is the local potential well in the galactic plane, the higher is the local accretion rate of the external gas, and the higher accretion rate provides more efficient star formation. The middle plot of Fig. 3 compares the $\mathrm{Mg} / \mathrm{Fe}$ ratios and the ages of the stellar systems. We can see the linear envelope on the right (the dotted line), where most of our galaxies concentrate. These are probably the stellar systems that have begun their star formation together in the early Universe, at $z=2-3$, and have finished it differently. Those that had finished it quickly have a higher average age of the stellar populations and a higher $\mathrm{Mg} / \mathrm{Fe}$ ratio, while those where the star formation continued for many billions of years evolved to the solar $\mathrm{Mg} / \mathrm{Fe}$ ratio. However, the point distribution in the middle panel of Fig. 3 has an amorphous tail to the left of the dotted line, and this tail comprises both the discs and the bulges. These are stellar systems where the last star formation episode took place later than commonly in the studied population of galaxies. Indeed, in order to have both $[\mathrm{Mg} / \mathrm{Fe}]=+0.2$ and the average stellar population age of 1.5-3 Gyr, they needed to undergo their only 1.0-1.5 Gyr of active star formation much later than at the redshift of $z=0.5$. Therefore, it turns out that the star formation episodes in the structural components - the discs and the bulges - of isolated lenticular galaxies might take place at different times and have various durations.

And finally, a few words about the lenses in the S0galaxies, which are usually thought to be genetically related with former starforming rings and dissolved bars. Until now, the common point of view was that stellar populations in lenses are old and that the lenses are dynamically hot (Kormendy 1984; Laurikainen et al. 2013). However, this point of view is based on a study of a few objects. In our small sample we were able to determine the properties of the stellar populations in seven lenses and old rings. We can certainly conclude that the lenses we have studied represent disc structures. On average, they have the same velocity dispersions as the surrounding discs (the left panel of Fig. 4). When we look at the individual velocity dispersion profiles, some local peaks can be noted within the area dominated by the lenses, for instance, in NGC 3098, NGC 6798, and UGC 4551; however, after averaging across the entire lens-dominated region, the peaks do not substantially contribute to the resulting averaged lens velocity dispersion, because the peaks surpass the velocity dispersion in the disc by no more than 20-30 $\mathrm{km} \mathrm{s}^{-1}$ which is comparable to the typical velocity dispersion measurement error. The charac- 
teristics of the stellar populations in the lenses/rings are generally the same as in the discs. We have found a single lens, located in UGC 4551, which is considerably younger than the surrounding disc. At the same time the lenses of intermediate age, 3-5 Gyr old, that do not differ by their stellar population parameters from the discs, were found in five galaxies.

Concerning the dynamical status of the central bulges, our small sample of isolated lenticular galaxies proved to have practically equal numbers of pseudobulges possessing the same stellar velocity dispersion as their discs, and of classical bulges which are dynamically hotter than the discs (the right panel in Fig. 4). This once again confirms that bulges in lenticular galaxies can be very different if regarding their luminosity and the contribution to the total mass of the galaxy or their origin and evolution. And this point is true even if we consider a sample of isolated lenticular galaxies, for which the influence of the environment on the evolution seems to be minimized.

As a summary, the results obtained for the stellar components of the sample of isolated lenticular galaxies presented in this work have confirmed our long-standing suspicions about the influence of the environment's density on the evolution of galaxies. Namely, for isolated lenticular galaxies, unlike what is observed for the members of groups and clusters, there is no fixed epoch when the structural components form: they may start their star formation in the bulges or in the discs at a $z>2$ as well as only a billion years ago. The scatter of the mean (SSP-equivalent) stellar ages in the discs of S0 galaxies indeed increases with decreasing density of the environment Sil'chenko et al. (2012) and reaches its maximum among the isolated galaxies.

What may affect the morphological "fate" of a disc galaxy residing in rarefied surroundings? Why can it prove to become lenticular or spiral to the present epoch? Everything points to the regime of accretion of the cold external gas, which is known to fuel star formation in the discs of present spiral galaxies over past billions of years, and this regime is likely to be stochastic. A recent study on searching for faint companions of isolated galaxies (Karachentseva et al. 2011) has revealed an interesting statistical feature: in isolated lenticular galaxies the companions have systematically larger relative velocities with respect to their host galaxy than the companions of isolated spiral galaxies; moreover, the isolated lenticular galaxies have no companions whatsoever with the systemic velocity difference of less than $50 \mathrm{~km} \mathrm{~s}^{-1}$. Does this kinematical feature mean that the companions of lenticular galaxies cannot be accreted by their hosts in the nearest future, whereas for the companions of spiral galaxies the dynamical friction is enough to provide their continuous accretion? Let us reverse the argument: it is possible that the orbital structure of the group of faint companions may be any one, and those disc galaxies whose system of the companions is dynamically cold can obtain fuel for the star formation in their discs and become spirals, but those which happened to have a dy- namically hot orbital system of companions (or in the present epoch they have already "dropped" onto themselves all the companions that could be dropped) stay lenticulars. A similar hint was noticed by us during the analysis of ionized-gas rotation in the isolated disc galaxies. In half the isolated lenticular galaxies containing ionized gas, its visible rotation is counterwise with respect to the direction of the rotation of stars Katkov et al. 2013). This may mean that the gas was accreted from the orbits isopropically inclined to the plane of the main stellar disc; while in spiral galaxies the gas with the "decoupled" kinematics is much more rare. This might imply that a steady accretion confined to the main stellar disc plane provides stable smooth accumulation of the cold gas suitable for continuous star formation, while the gas infall from inclined orbits leads certainly to developing gas turbulence, heating, and so preventing the star formation ignition. For instance, generation of shock waves during the passage of the potential well of the galactic disc by a polar-ring gas was described by Wakamatsu (1993). Or shock waves may be generated during gas accretion through the collision of the external gas with the primary gas of the galaxy, already existing in the disc. This effect, depending on the geometry of accretion of the external gas, may also affect the shaping of the morphological type of a galaxy.

\section{CONCLUSIONS}

In this paper we have presented the results of long-slit spectroscopy for a sample of isolated lenticular galaxies. As a result of observations with the SCORPIO-2 and SCORPIO reducers of the 6-meter BTA telescope of the SAO RAS, we have measured radial profiles of the stellar rotation velocity, stellar velocity dispersion, the SSP-equivalent ages and the SSP-equivalent metallicities of stars in 12 objects. One of them, IC 875, has proved to be a low-luminosity elliptical galaxy. We have analyzed the statistics of the characteristics obtained for the radially resolved stellar populations, by basing on the data of the remaining 11 galaxies. The ages of the stellar populations in our sample of isolated lenticular galaxies cover the full range of values, from 1.5 to 15 Gyr, and, unlike the S0 galaxies in denser environments, the isolated galaxies tend to have the same ages of stars in the bulges and discs; obviously, they had no chance of separate bulge rejuvenation. The lenses and rings of enhanced stellar brightnesses, detected on radial brighntness profiles in 7 of 11 galaxies, usually have the stellar populations and the stellar velocity dispersions indistinguishable from the properties of the discs. We suggest that probably the shaping of the morphological type of disc galaxies in complete isolation critically depends on the possible regimes (continuity and geometry) of accretion of external cold gas. 


\section{Acknowledgments}

The authors thank Dmitry Makarov for his help with the database of galaxies of the Local Volume and construction of the sample of isolated lenticular galaxies, and Nikolay Borisov for his support with the SCORPIO2 observations at the BTA. During the analysis of the sample galaxies we have used the Lyon-Meudon extragalactic database (HyperLeda), supported by the LEDA team at the Lyon Observatory CRAL (France), and the database of extragalactic data NASA/IPAC (NED), managed by the the Jet Propulsion Laboratory of the California Institute of Technology under a contract with National Aeronautics and Space Administration (NASA, USA). As a source of photometric data, we have used the public archives of the SDSS-III project (http://www.sdss3.org), supported by the Alfred P. Sloan foundation, the institutions participating in the SDSS collaboration, the National Science Foundation (NSF), and the U.S. Department of Energy, as well as the data of the Two Micron All Sky Survey (2MASS), which was jointly performed by the University of Massachusetts and the infrared data analysis center at Caltech with the financial support from the NASA and NSF. Our study of isolated lenticular galaxies was supported by the RFBR grants No. 13-02-00059a and 12-02-00685a. I. Katkov expresses gratitude to the nonprofit Dynasty foundation for supporting his research. The observations on the 6-meter BTA telescope were held with the financial support of the Ministry of Education and Science of the Russian Federation (state contracts No. 14.518.11.7070, 16.518.11.7073)

\section{REFERENCES}

Afanasiev, V. L., \& Moiseev, A. V. 2005, Astronomy Letters, 31,194

-. 2011, Baltic Astronomy, 20, 363

Barway, S., Mayya, Y. D., Kembhavi, A. K., \& Pandey, S. K. 2005, Astron. J, 129, 630

Bekki, K., \& Couch, W. J. 2011, Monthly Notices Roy. Astron. Soc, 415, 1783

Birnboim, Y., Dekel, A., \& Neistein, E. 2007, Monthly Notices Roy. Astron. Soc, 380, 339

Byrd, G., \& Valtonen, M. 1990, Astrophys. J, 350, 89

Cappellari, M., \& Emsellem, E. 2004, PASP, 116, 138

Cappellari, M., Emsellem, E., Krajnović, D., et al. 2011, Monthly Notices Roy. Astron. Soc, 416, 1680

Chilingarian, I., Prugniel, P., Sil'Chenko, O., \& Koleva, M. 2007a, in IAU Symposium, Vol. 241, IAU Symposium, ed. A. Vazdekis \& R. Peletier, 175-176

Chilingarian, I. V., Prugniel, P., Sil'Chenko, O. K., \& Afanasiev, V. L. 2007b, Monthly Notices Roy. Astron. Soc, 376,1033

Davies, R. L., Efstathiou, G., Fall, S. M., Illingworth, G., \& Schechter, P. L. 1983, Astrophys. J, 266, 41

Davis, T. A., Alatalo, K., Sarzi, M., et al. 2011, Monthly Notices Roy. Astron. Soc, 417, 882

Dressler, A. 1980, Astrophys. J, 236, 351

Gunn, J. E., \& Gott, III, J. R. 1972, Astrophys. J, 176, 1

Hubble, E. P. 1936, Realm of the Nebulae

Icke, V. 1985, Astron. and Astrophys, 144, 115
Jones, L. A., \& Worthey, G. 1995, Astrophys. J. Let, 446, L31

Karachentsev, I. D., \& Makarov, D. I. 2008, Astrophysical Bulletin, 63, 299

Karachentsev, I. D., Makarov, D. I., Karachentseva, V. E., \& Melnyk, O. V. 2011, Astrophysical Bulletin, 66, 1

Karachentseva, V. E., Karachentsev, I. D., \& Melnyk, O. V. 2011, Astrophysical Bulletin, 66, 389

Katkov, I. Y., \& Chilingarian, I. V. 2011, in Astronomical Society of the Pacific Conference Series, Vol. 442, Astronomical Data Analysis Software and Systems XX, ed. I. N. Evans, A. Accomazzi, D. J. Mink, \& A. H. Rots, 143

Katkov, I. Y., Sil'chenko, O. K., \& Afanasiev, V. L. 2013, Monthly Notices Roy. Astron. Soc

Kormendy, J. 1984, Astrophys. J, 286, 116

Kormendy, J., \& Bender, R. 2012, Astrophys. J. Suppl, 198, 2

Kronberger, T., Kapferer, W., Ferrari, C., Unterguggenberger, S., \& Schindler, S. 2008, Astron. and Astrophys, 481, 337

Larson, R. B., Tinsley, B. M., \& Caldwell, C. N. 1980, Astrophys. J, 237, 692

Laurikainen, E., Salo, H., Athanassoula, E., et al. 2013, Monthly Notices Roy. Astron. Soc, 430, 3489

Laurikainen, E., Salo, H., Buta, R., Knapen, J. H., \& Comerón, S. 2010, Monthly Notices Roy. Astron. Soc, 405, 1089

Le Borgne, D., Rocca-Volmerange, B., Prugniel, P., et al. 2004, Astron. and Astrophys, 425, 881

MacArthur, L. A., Courteau, S., \& Holtzman, J. A. 2003, Astrophys. J, 582, 689

Makarov, D., \& Karachentsev, I. 2011, Monthly Notices Roy. Astron. Soc, 412, 2498

Makarov, D. I., \& Karachentsev, I. D. 2009, Astrophysical Bulletin, 64, 24

Matteucci, F., \& Greggio, L. 1986, Astron. and Astrophys, 154,279

Méndez-Abreu, J., Aguerri, J. A. L., Corsini, E. M., \& Simonneau, E. 2008, Astron. and Astrophys, 478, 353

Moore, B., Katz, N., Lake, G., Dressler, A., \& Oemler, A. 1996, Nature, 379, 613

Naim, A., Lahav, O., Buta, R. J., et al. 1995, Monthly Notices Roy. Astron. Soc, 274, 1107

Prugniel, P., \& Koleva, M. 2012, in IAU Symposium, Vol. 284, IAU Symposium, ed. R. J. Tuffs \& C. C. Popescu, 16-19

Prugniel, P., Soubiran, C., Koleva, M., \& Le Borgne, D. 2007, ArXiv Astrophysics e-prints, arXiv:astro-ph/0703658

Quilis, V., Moore, B., \& Bower, R. 2000, Science, 288, 1617

Salpeter, E. E. 1955, Astrophys. J, 121, 161

Sancisi, R., Fraternali, F., Oosterloo, T., \& van der Hulst, T. 2008, A\&A Rev., 15, 189

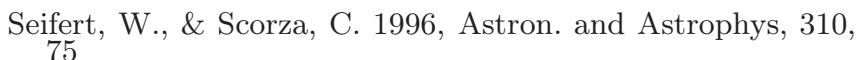
Sil'Chenko, O. K., Chilingarian, I. V., Sotnikova, N. Y., \& Afanasiev, V. L. 2011, Monthly Notices Roy. Astron. Soc, 414,3645

Sil'chenko, O. K., Proshina, I. S., Shulga, A. P., \& Koposov, S. E. 2012, Monthly Notices Roy. Astron. Soc, 427, 790

Simien, F., \& Prugniel, P. 2002, Astron. and Astrophys, 384, 371

Spitzer, Jr., L., \& Baade, W. 1951, Astrophys. J, 113, 413

Sulentic, J. W., Verdes-Montenegro, L., Bergond, G., et al. 2006, Astron. and Astrophys, 449, 937

Thomas, D., Maraston, C., \& Bender, R. 2003, Monthly Notices Roy. Astron. Soc, 339, 897 
Tinsley, B. M. 1979, Astrophys. J, 229, 1046

Trager, S. C., Faber, S. M., Worthey, G., \& González, J. J. 2000, Astron. J, 120, 165

van den Bergh, S. 1976, Astrophys. J, 206, 883 van der Marel, R. P., \& Franx, M. 1993, Astrophys. J, 407,

van Dokkum, P. G. 2001, PASP, 113, 1420

Wakamatsu, K.-I. 1993, Astron. J, 105, 1745

Walcher, C. J., Coelho, P., Gallazzi, A., \& Charlot, S. 2009, Monthly Notices Roy. Astron. Soc, 398, L44

Worthey, G., Faber, S. M., Gonzalez, J. J., \& Burstein, D. 1994, Astrophys. J. Suppl, 94, 687

Zasov, A. V., Sil'chenko, O. K., Katkov, I. Y., \& Dodonov, S. N. 2013, Astronomy Letters, 39, 17 